\title{
Results from a recent stellar rotation census of B stars
}

\author{
Wenjin Huang ${ }^{1}$, Douglas R. Gies ${ }^{2}$ and M. Virginia McSwain ${ }^{3}$ \\ ${ }^{1}$ Department of Astronomy, University of Washington, Box 351580, Seattle, WA 98195-1580 \\ email: hwenjin@astro. washington.edu \\ ${ }^{2}$ Center for High Angular Resolution Astronomy, Department of Physics and Astronomy \\ Georgia State University, P.O. Box 4106, Atlanta, GA 30302-4106; \\ email: gies@chara.gsu.edu \\ ${ }^{3}$ Department of Physics, Lehigh University, 16 Memorial Drive East, Bethlehem, PA 18015; \\ email: mcswain@lehigh.edu
}

\begin{abstract}
In an analysis of the rotational properties of more than $1100 \mathrm{~B}$ stars ( 660 cluster and $\sim 500$ field $\mathrm{B}$ stars), we determine the projected rotational velocity ( $\mathrm{V} \sin \mathrm{i}$ ), effective temperature, gravity, mass, and critical rotation speed for each star. The new data provide us a solid observational base to explore many hot topics in this area: Why do field B stars rotate slower than cluster B stars? How fast do B stars rotate when they are just born? How fast can $\mathrm{B}$ stars rotate before they become Be stars? How does the rotation rate of B stars change with time? Does the evolutionary change in rotation velocity lead to the Be phenomenon? Here we report the results of our efforts in searching for answers to these questions based on the latest B star census.
\end{abstract}

Keywords. line: profiles, stars: early-type, stars: emission-line, Be, stars: fundamental parameters, stars: rotation

\section{Observation and Analysis}

Modern theoretical studies (Heger \& Langer 2000; Meynet \& Maeder 2000) predict that stellar rotation strongly influences the evolution of massive OB stars. Rapid rotation can trigger strong interior mixing, extend the core hydrogen-burning lifetime, significantly alter the luminosity, and change the chemical composition of the stellar surface over time. Spectroscopic investigation on a large and homogeneous star sample can provide the key data for us to measure rotational properties of massive stars and their evolutionary state and, therefore, is an ideal way to test our knowledge of the interior structure of a massive rotating star. Our first spectroscopic survey was carried out for about 500 cluster B stars in 2000 and 2001 (Huang \& Gies 2006a). More recently, we accomplished two similar spectroscopic observing campaigns in 2006 (obtained $\sim 230$ cluster B stars) and in 2008 (obtained $\sim 370$ field B stars). In addition to these observed stars, about 100 field B stars from the Indo-U. S. stellar spectra Library are also included in our analysis.

For each star in our sample, we derived $V \sin i$ from a line profile fit using the realistic models with the gravitational darkening effect in mind. Then we determined $T_{\text {eff }}$ and $\log g$ values by fitting the $\mathrm{H} \gamma$ profile, and estimated $\log g_{\text {polar }}$ which is not affected by stellar rotation and, therefore, can be used as a good indicator of evolutionary status of a rotating star. We also estimated stellar mass according to $T_{\text {eff }}$ and $\log g_{\text {polar }}$, and we then calculated the critical velocity, $V_{\text {crit }}$, for each star. The details of these steps can be found in Huang \& Gies (2006a,b) and Huang, Gies \& McSwain (2010). Among the 1100 B stars, we found about 160 radial velocity variables which were not used to derive the statistical results summarized below. 


\section{Results}

We summarize our major findings below:

1) The mean $V \sin i$ for the field sample (441 stars) is slower than that for the cluster sample (557 stars), confirming results from previous studies (Abt, Levato, \& Grosso 2002; Huang \& Gies 2006a; Wolff et al. 2007). By comparing stars with similar evolutionary status, we find that the stars in these two samples have similar rotational properties when plotted as a function of $\log g_{\text {polar }}$. Thus we conclude that the overall slowness of rotation of field $\mathrm{B}$ stars is mainly due to the presence of a larger fraction of more evolved stars than found among cluster B stars.

2) The rotation distribution curves based on young stars with $\log g_{\text {polar }}>4.15$ suggest that massive stars are born at various rotation rates, including some very slow rotators $\left(V_{\text {eq }} / V_{\text {crit }}<0.1\right)$. The mass dependence suggests that higher mass B stars may preferentially experience angular momentum loss processes during and after formation. The low mass stars are born with more rapid rotators than high mass stars if the stellar rotation rate is evaluated by $V_{\text {eq }} / V_{\text {crit }}$.

3) The statistics based on the normal B (non-Be) stars in our sample indicates that low mass B stars $\left(<4 M_{\odot}\right)$ may require a high threshold of $V_{\text {eq }} / V_{\text {crit }}>0.96$ to become Be stars. As stellar mass increases, this threshold decreases, dropping to 0.64 for B stars with $M>8.6 M_{\odot}$. This implies that the mass loss processes leading to disk formation may be very different for low and high mass Be stars.

4) Comparing with modern evolutionary models of rotating stars (for 3 and $9 M_{\odot}$ from Ekström et al. 2008), the apparent evolutionary trends of $\left\langle V \sin i / V_{\text {crit }}\right\rangle$ are in good agreement for the high mass B stars, but the data for low mass B stars shows a more pronounced spin-down trend than predicted.

5) Predictions for the fractions of rapid rotators and Be stars produced by the redistribution of angular momentum with evolution agree with observations for the higher mass B stars but vastly overestimate the Be population for the lower mass stars. The greater than expected spin-down of the lower mass stars explains this discrepancy and suggests that most of the low mass Be stars were probably spun up recently.

\section{Acknowledgments}

This material is based upon work supported by the National Science Foundation grant AST-0606861 (DRG). WJH thanks G. Wallerstein and the Kenilworth Fund of the New York Community Trust for partial financial support of this study. WJH is very grateful for partial finance support from NSF grant AST-0507219 to J. G. Cohen. MVM is grateful for support from NSF grant AST-0401460 as well as Lehigh University. This research has made use of the Simbad database, operated at CDS, Strasbourg, France, and of the Webda database, operated at the Institute for Astronomy of the University of Vienna.

\section{References}

Abt, H. A., Levato, H., \& Grosso, M. 2002, ApJ, 573, 359

Ekström, S., Meynet, G., Maeder, A., \& Barblan, F. 2008, A\&SA, 478, 467

Heger, A. \& Langer, N. 2000, ApJ, 544, 1016

Huang, W. \& Gies, D. R. 2006a, ApJ, 648, 580

Huang, W. \& Gies, D. R. 2006b, ApJ, 648, 591

Huang, W., Gies, D. R., \& McSwain, M. V. 2010, ApJ 722, 605

Meynet, G. \& Maeder, A. 2000, A\&\&A, 361, 101

Wolff, S. C., Strom, S. E., Dror, D., \& Venn, K. 2007, AJ, 133, 1092 